\title{
RELAÇÕES INTERORGANIZACIONAIS: PERSPECTIVA SOCIOLÓGICA PARA ANÁLISE DOS MICROPROCESSOS
}

Anelise Rebelato Mozzato ${ }^{1}$

Luiz Fernando Fritz Filho ${ }^{1}$

${ }^{1}$ Universidade de Passo Fundo 


\section{Relações interorganizacionais: perspectiva sociológica para análise dos microprocessos}

\section{Resumo:}

Este ensaio teórico tem como objetivo refletir sobre as relações interorganizacionais, a partir da perspectiva sociológica, contribuindo com o debate sobre a necessidade de considerar racionalidades alternativas à instrumental nos estudos sobre relações inteorganizacionais, resgatando-se pressupostos teóricos críticos dos estudos organizacionais e da estratégia organizacional. Nessa lógica, parte-se da ideia de que as relações interorganizacionais se dão no cotidiano, por meio das interações entre os diferentes agentes, considerando como importante a análise dos microprocessos, como inerentes e complementares a análises em nível meso e macro. A justificativa é a de que a racionalidade instrumental inerente à Ciência Social dominante no Ocidente, necessita de alternativas e novas possibilidades ao mainstream. Portanto, salienta-se a necessidade de se considerar racionalidades alternativas à instrumental nas estratégias interorganizacionais, em favor de uma competitividade sustentável, a qual busca um olhar para além do utilitarismo econômico nas relações interorganizacionais.

Palavras-chave: Relações interorganizacionais. Perspectiva sociológica. Microprocessos. 


\section{Introdução}

Por mais que os estudos sobre cooperação entre firmas tenham despontado no final do século XX como alternativa estratégica no Brasil, é na primeira década do século XXI que surgem contribuições teóricas para verificação empírica. No entanto, a maior parte dos estudos, sobre relações de cooperação interorganizacional, segue a racionalidade instrumental da visão dominante, do mainstream econômico. Todavia, alguns estudos brasileiros apontam para a necessidade de se pensar para além da visão instrumental, apresentando sugestões inerentes a outras perspectivas teóricas. Em nível internacional, Crooper, Ebers, Huxham e Ring (2014) apontam para essa necessidade, salientando que os estudos devem ser enriquecidos por diferentes concepções teóricas, como a sociológica, psicológica, política, evolucionária e crítica. No Brasil pesquisas empíricas desenvolvidas por Tureta e Lima (2011), Vizeu, Guarido Filho e Gomes (2014) e Balestrin, Verschoore e Perucia (2014) podem ser citadas como exemplos de estudos que se valeram de outras perspectivas de análise.

Diante do entendimento da necessidade de se debater as relações interorganizacionais sob outras lentes, este ensaio teórico tem como objetivo refletir sobre as relações interorganizacionais a partir da perspectiva sociológica, contribuindo com o debate sobre a necessidade de considerar racionalidades alternativas à instrumental nos estudos sobre relações inteorganizacionais, resgatando-se pressupostos teóricos críticos dos estudos organizacionais e da estratégia organizacional. Assim, assume-se a visão relacional da estratégia, partindo da ideia de que as relações interorganizacionais se dão no cotidiano, por meio das interações entre os diferentes agentes que mantém interesses similares e também antagônicos.

Nessa lógica, os questionamentos, o pensamento crítico, a dialética e o pensar a partir da visão relacional da estratégia, no sentido proposto por Whittington (2006), Johnson et al. (2007), Tureta e Lima (2011), Crooper, Ebers, Huxham e Ring (2014), Balestrin, Verschoore e Perucia (2014) e Vizeu, Guarido Filho e Gomes (2014), fazem-se necessários. Apesar do debate nacional e internacional já terem avançado, em torno da perspectiva sociológica para análise das relações intereorganizacionais, percebe-se que as correntes funcionalistas continuam predominando, sendo trabalhadas na visão utilitarista da vantagem competitiva de Porter (1998), visando atingir os fins meramente econômicos.

Sob a visão relacional da perspectiva sociológica, temas emergentes que perpassam as relações interorganizacionais necessitam de debate mais aprofundado, a exemplo da confiança, interdependência, conflitos, capital social, poder, gestão do conhecimento e Aprendizagem Interorganizacional (AIO). A análise desses microprocessos, sem a negação do nível meso e macro, sob a lente teórica da sociologia, pode elucidar e facilitar o olhar macro nas relações interorganizacionais estabelecidadas em diferentes espaços que mantém a cooperação interorganizacional.

Os resultados dessas reflexões são apresentados a seguir, iniciando-se o debate sobre as relações interorganizacionais e a predominância da perspectiva funcionalista (racionalidade instrumental) nos estudos sobre o tema. Na sequência, trabalha-se a perspectiva sociológica como uma possibilidade alternativa ao mainstream, salientando-se os microprocessos nas relações interorganizacionais. Portanto, diante da alternativa de perspectiva que vai além do olhar puramente econômico, apresenta-se a possibilidade alternativa a racionalidade

\section{Organizadores:}

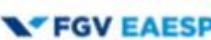

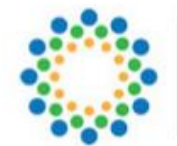
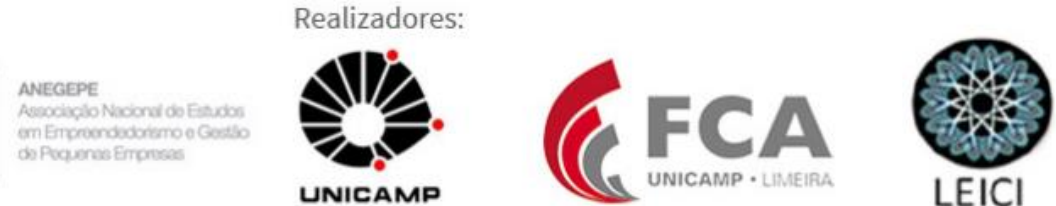
instrumental (visão utilitarista). Por fim, são delineadas as considerações finais, com indicação de pesquisas futuras.

\section{Estudos das relações interorganizacionais: da perspectiva funcionalista para a sociológica}

A predominância da racionalidade instrumental, perspectiva funcionalista, nos estudos das relações interorganizacionais não pode ser negada. Como referem Mozzato e Grzybovski (2013, p.506):

percebe-se que o funcionalismo apresenta pressupostos teóricos relacionados à regulação e à objetividade orientando-se pelos métodos das ciências naturais para compreender os indivíduos e se apresentando como racionalista e pragmático, com foco na estrutura, baseado em uma ordem social regulada. Nesse sentido, busca prover soluções práticas e objetivas calcadas no determinismo.

Assim, tradicionalmente, também a área de estratégia tem sido estudada com esta perspectiva, principalmente, com um olhar meramente econômico. Isto posto, Tenório (2004) propõe a renovação na gestão, contrapondo-se ao enfoque funcionalista e à racionalidade instrumental. Aktouf (1996) já defendia a necessidade da "administração renovada", dentre tantos outros teóricos críticos brasileiros, a exemplo de Alberto Guerreiro Ramos, José Henrique Faria, Fernando Prestes Motta e Maurício Tragtenberg.

Diante da necessidade primordial de um modelo de competitividade sustentável, é preciso um outro olhar sobre as estratégias organizacionais, em que não predomine a ortodoxia. Assim, a teoria crítica é uma abordagem alternativa ao modo de gerar conhecimento, podendo trazer avanços também aos estudos sobre as relações interorganizacionais, constituindo-se em um dos caminhos possíveis na direção de uma sociedade melhor e mais justa. "Há concepções epistemológicas alternativas ao funcionalismo ortodoxo, especialmente, racionalidades que se impõem e devem ser consideradas e pensadas como possíveis na perspectiva de mudanças no fazer ciência com responsabilidade em relação ao ser humano e à sociedade" (MOZZATO; GRZYBOVSKI, 2013, p.516).

Nessa lógica, na perspectiva da Estratégia como Prática Social (EPS), a estratégia é analisada como algo que as pessoas fazem (JOHNSON et al., 2007). Nessa linha da virada para a prática, a perspectiva da estratégia como prática admite que o contexto social assume importância ímpar. Segundo Whittington (2006) e Jarzabkowski, Balogun e Seidl (2007), a análise da estratégia precisa considerar cada pessoa, as práticas de cada organização, as atividades (práxis) da estratégia e o contexto social.

Assim, Tureta e Lima (2011) trabalham o conceito de redes interorganizacionais partindo do estrutural para o relacional, seguindo a EPS, tendo como foco as práticas cotidianas, adotando a abordagem de Whittington (2006) e Johnson et al. (2007) no campo da estratégia. "A EPS se apresenta como uma alternativa às perspectivas macro que relegavam a dimensão micro ao segundo plano e não consideravam como centrais as relações entre as pessoas e suas práticas desempenhadas no cotidiano das organizações" (TURETA; LIMA, 2011, p.78). Na lógica sociológica, como bem pontua Pires (2011), os territórios precisam ser entendidos em sua realidade particular própria, com suas demandas legítimas.
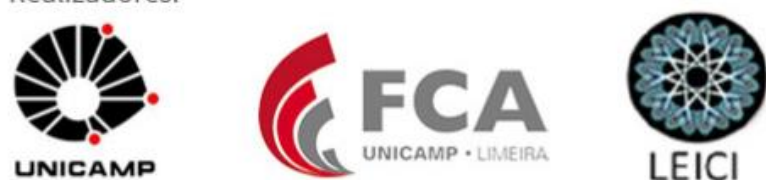


\section{Nível de análise interorganizacional: perspectiva sociológica}

As relações interorganizacionais compreendidas pela visão relacional da estratégia têm como foco a cooperação, não a competição, mesmo não se negando a "coopetição" (NALEBUFF; BRANDENBURGER, 1996). É uma visão que vai além do olhar econômico nas relações interorganizacionais, da visão utilitarista que tem como base Porter (1998), constituindo-se no mainstream econômico.

No que tange a perspectiva sociológica da estratégia em organizações, Kirschbaum e Guarido Filho (2011, p.17) pontuam que "os aspectos econômicos da ação estratégica ganham outros contornos." No Quadro 1, os autores apresentam o comparativo da perspectiva sociológica e a do mainstream econômico.

Quadro 1 - Comparativo entre a perspectiva sociológica e o mainstream econômico COMPARAÇÃO ENTRE PERSPECTIVA SOCIOLÓGICA E MAINSTREAM ECONÔMICO

\begin{tabular}{|c|c|c|}
\hline & PERSPECTIVA SOCIOLOGICA & MAINSTREAM ECONÔMICO \\
\hline Concepçáo de ator & $\begin{array}{l}\text { Influenciado por outros atores e } \\
\text { integrante de grupos e sociedade. }\end{array}$ & Ator năo influenciado por outros. \\
\hline $\begin{array}{l}\text { Concepçăo de } \\
\text { organizaçăo }\end{array}$ & $\begin{array}{l}\text { Produtos históricos; possuem } \\
\text { estruturas formais e informais; } \\
\text { envolvem múltiplos atores. }\end{array}$ & $\begin{array}{l}\text { Soluçōes instrumentais para } \\
\text { problemas; hierarquias com alguns } \\
\text { atores convencionais. }\end{array}$ \\
\hline Açāo econômica & $\begin{array}{l}\text { Diferentes tipos de açōes } \\
\text { econômicas; racionalidade é } \\
\text { variável. }\end{array}$ & $\begin{array}{l}\text { Todas as açōes econômicas } \\
\text { sáo consideradas racionais; } \\
\text { racionalidade é pressuposto. }\end{array}$ \\
\hline $\begin{array}{l}\text { Condicionantes da } \\
\text { açáo econômica }\end{array}$ & $\begin{array}{l}\text { Recursos, estrutura social e } \\
\text { estruturas de significado. }\end{array}$ & $\begin{array}{l}\text { Preferências, escassez de recursos } \\
\text { e tecnologia. }\end{array}$ \\
\hline $\begin{array}{l}\text { Economia e } \\
\text { sociedade }\end{array}$ & $\begin{array}{l}\text { Economia é parte da sociedade; } \\
\text { sociedade é a base de referência. }\end{array}$ & $\begin{array}{l}\text { Mercado e a economia săo as } \\
\text { bases de referência; sociedade é } \\
\text { um dado. }\end{array}$ \\
\hline $\begin{array}{l}\text { Relação da } \\
\text { organizaçăo com o } \\
\text { ambiente }\end{array}$ & $\begin{array}{l}\text { Organizaçōes são parte de um } \\
\text { contexto mais amplo (campos, } \\
\text { sociedade). }\end{array}$ & $\begin{array}{l}\text { Organizaçōes isoladas interagem } \\
\text { com outras por meio de contatos } \\
\text { instrumentais. }\end{array}$ \\
\hline
\end{tabular}

Fonte: Kirschbaum e Guarido Filho (2011, p.17)

A visão relacional da estratégia (perspectiva sociológica), na lógica apontada por Kirschbaum e Guarido Filho (2011), tem ganho espaço no campo da estratégia, inclusive no nível de análise interorganizacional, como pode ser observado nos trabalhos de Balestrin, Verschoore e Perucia (2014) e Vizeu, Guarido Filho e Gomes (2014), entre outros. Estes últimos apontam para o olhar além do econômico também no nível interoganizacional. Complementarmente, Kirschbaum (2015) afirma que as redes interorganizacionais podem ser inclusivas.

Portanto, não se trata da negação do aspecto econômico, mas sim, da rejeição da exploração econômica intensiva e insustentável. Para tanto, vários aspectos precisam ser 
respeitados de fato, a exemplo da cultura local, regional e nacional, como bem pontuam Matos, Amaral Filho e Costa (2017). Os autores fazem referência a Celso Furtado quanto ao fato de que "o desenvolvimento substantivo passa pelo cultivo e pelo fortalecimento dos valores da própria cultura" (MATOS; AMARAL FILHO; COSTA, 2017, p.233). Em sentido semelhante, estudos como os de Szapiro, Lemos, Lastres, Cassiolato e Vargas (2017) destacam a importância de mais estudos na lógica da competitividade sustentável. No que tange a realidade brasileira e latino-americana afirmam que estudos devem focar na importância do pensar e implementar estratégias de desenvolvimento que não sejam subordinadas a realidade internacional. Tais estudos destacam a importância dos relacionamentos cooperativos.

Crooper, Ebers, Huxham e Ring (2014) assinalam alguns temas nos quais os estudos sobre relações interorganizacionais precisam avançar, sendo eles: confiança, capital social, poder, mudanças e temporalidade. Nessa lógica, a dimensão dos microprocessos torna-se central, lançando olhar sobre as relações entre as pessoas, possibilitando assim pesquisar como e porque os eventos acontecem, com as consequências respectivas. Admite-se assim o constante estado de transformação.

\subsection{Análise dos microprocessos nas relações interorganizacionais - na lógica relacional}

$\mathrm{Na}$ lógica da visão relacional, a sociedade torna-se a base de referência, como tal, as mudanças nas relações são impactadas de acordo com os movimentos do global e do local. Assim, as práticas individuais e coletivas refletem e são refletidas nas relações interorganizacionais. "A estratégia coletiva cria uma fonte inimitável de recursos através de uma rede com acesso valioso a informações, compartilhamento de conhecimento, complementaridade de recursos, investimentos específicos de relacionamento e governança efetiva" (BALESTRIN; VERSCHOORE; PERUCIA, 2014, p.51).

Portanto, com base nos temas de estudos apontados como emergentes por Crooper, Ebers, Huxham e Ring (2014) nas relações interorganizacionais, entende-se que os microprocessos que envolvem a interdependência colaborativa, a AIO e as trocas de conhecimento precisam ser mais estudados e podem ser mais bem entendidos sob a visão relacional e não substantiva da estratégia, e ainda, relacionado aos meso e macroprocessos.

$\mathrm{Na}$ lógica da racionalidade substantiva preconizada pela visão sociológica, a AIO e a troca de conhecimentos são facilitadas, ainda mais quando se considera a interdependência colaborativa $^{1}$, pois esta exige atitudes coletivas e comprometidas, prevalecendo o objetivo coletivo (NOHRIA; ECLES, 1992; RUSBULT; KUBACKA, 2009).

A interdependência tanto de objetivos quanto de recursos ou complementaridade na realização de tarefas revela compromisso recíproco existente entre os parceiros que mantém relações interorganizacionais (LUBATKIN; FLORIN; LANE, 2001; MUTHUSAMY;

\footnotetext{
${ }^{1}$ Conforme Lubatkin, Florin e Lane (2001) e Muthusamy e White (2005), a interdependência diz respeito à vinculação entre os diferentes agentes, podendo ser, tanto referente aos objetivos (objetivos comuns entre os diferentes agentes, evidenciando interesses convergentes), como aos recursos (interdependência de recursos entre os agentes e refere-se à partilha ou utilização conjugada de recursos, havendo receptividade e trocas em razão das necessidades para a realização de dado negócio) e na complementaridade na realização das tarefas (interdependência entre os agentes no que tange a realização de tarefas conjuntas, havendo receptividade e trocas no sentido de complementar as tarefas).
}

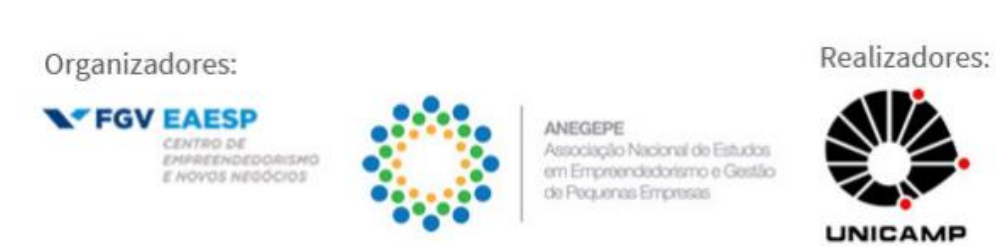

UNICAMP

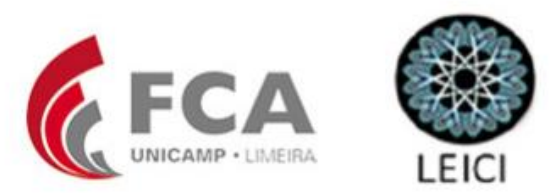


WHITE, 2005), consolidando assim a interdependência colaborativa. Como pontuam Human e Provan (1997) e Muthusamy e White (2005), as informações fazem parte da interdependência e o parceiro necessita estar receptivo a elas e a aprender, o que influencia positivamente na capacidade de absorção, culminando na suscetibilidade para o aprendizado.

Autores internacionais (CROSSAN; LANE; WHITE; DJURFELDT, 1995; GREVE, 2005; INKPEN; TSANG, 2007; NOOTEBOON, 2008; CROSSAN; MAUER; WHITE, 2011; GIBB; SUNE; ALBERS, 2017) e nacionais (ESTIVALETE; PEDROZO; CRUZ, 2008; BALESTRIN; VERSCHOORE, 2008; MOZZATO; BITENCOURT, 2014) apontam que a AIO é tema emergente que necessita de ampliação e aprofundamento. Como assinalam Mozzato e Bitencourt (2014), seguindo a visão relacional da estratégia, a AIO é entendida como parte de um contínuo de aprendizagem organizacional e é analisada sob uma abordagem menos cognitiva e mais social-comportamental. Ou seja, a aprendizagem é vista como um processo e os diferentes níveis que ocorrem dentro desse processo, sendo analisada sob a abordagem baseada na prática (perspectiva sociológica), seguindo os pressupostos de Silvia Gherardi.

$\mathrm{Na}$ perspectiva sociológica o processo da aprendizagem é socialmente construído na medida em que o indivíduo interage nas atividades cotidianas (STRATI, 2007; GHERARDI, 2009a; 2009b; 2014). A aprendizagem acontece a partir da participação das pessoas em atividades sociais, e a reflexividade está diretamente ligada à participação, ocorrendo no fluxo das experiências do dia a dia (GHERARDI; NICOLINI, 2001). Nessa lógica, o conhecimento é resultado da contínua construção, das conexões em ação, do conhecer e o fazer na participação (GHERARDI, 2000). Ou seja, o conhecer não se encontra separado do saber (ANTONELLO; AZEVEDO, 2011). Pontuam as autoras que a aprendizagem e o conhecimento são vistos como processos na ação das atividades das pessoas, assim percebidos de maneira mais crítica e analítica. Confere-se, portanto, evidência às realizações coletivas, resultado das interações entre os humanos e não humanos (BERTOLIN; CAPPELLE; BRITO, 2014; GHERARDI, 2014; 2015). Como bem pontua Bispo (2013), a perspectiva sociológica pressupõe que não há como as pessoas aprenderem algo que não esteja posto em um espaço social de interação, o que contrapõe a perspectiva mais pragmática da estratégia.

As práticas constituem-se em ações que acontecem no fluxo cotidiano das atividades, tanto em espaços sociais estruturados (situações formais) como não estruturados (informais/casuais), desencadeando a aprendizagem (JANOWICZ-PANJAITAN; NOORDERHAVEN, 2008; CORRADI et al., 2010; SANDBERG; TSOUKAS, 2011). Tanto em situações formais como informais de aprendizagem, a participação se dá por meio da interação entre indivíduos em diferentes espaços sociais, os quais envolvem comunicação, diálogo e reflexividade (LARSSON et al., 1998; MACDONALD; CROSSAN, 2010).

Um elemento central para que ocorra a aprendizagem é a existência de elos de confiança entre os envolvidos em relações interorganizacionais, ficando evidente que a mesma necessita ser gerada, desenvolvida e mantida (HIBBERT et al., 2010). Portanto, a confiança é vista como fator explicativo para as relações de cooperação, facilitando as relações de trocas (WOOLTHUIS; HILLEBRAND; NOOTEBOOM, 2005; BACHMANN; ZAHEER, 2008). Especialmente a confiança relacional, derivada de interações repetidas das partes ao longo do tempo, gera expectativas positivas sobre as intenções das partes (ROUSSAEU et al., 1998) e pode influenciar positivamente a cooperação. Além disso, o processo coletivo de aprendizagem
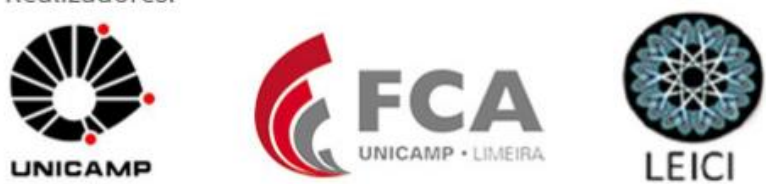
é fortalecido por relacionamentos de longo prazo baseados em confiança (LARSSON et al., 1998).

\section{Considerações finais}

Chegando-se ao final desse ensaio teórico, analisando o exposto entende-se como necessário recorrer a constructos e teorias ainda pouco consideradas pelo maistream da estratégia também no nível de análise interorganizacional. Assim, não se rejeita a abordagem econômica, mas circunscrevem-se pressupostos mais abrangentes, a exemplo da perspectiva sociológica, como afirmam Kirschbaum e Guarido Filho (2011). Nessa lógica, a sociedade é a base de referência. Assim, busca-se um olhar para além do utilitarismo econômico nas relações interorganizacionais, podendo essas serem inclusivas, como refere Kirschbaum (2015).

Nesse contexto, é preciso entender os territórios a partir da sua realidade particular própria, estudando os conflitos e os movimentos sociais inerentes, dessa forma, "mergulhando" no cenário real, tal como ele se apresenta. Assim, o foco fica direcionado para o social, onde é possível e necessário analisar os diferentes estágios de cada configuração interorganizacional, desde o surgimento, passando pelo fortalecimento, até chegar num estágio mais evoluído. Nesta linha os processos de governança territorial, através do estabelecimento de práticas democráticas locais, da participação de inúmeros atores em diferentes níveis e nos processos de decisão, reforçam mutuamente as demandas legítimas de acordo com as particularidades de cada local tornando-se uma importante referência e apoio para a perspectiva sociológica de análise (PIRES, 2011).

Além disso, tais análises vão passar pela questão da confiança e pela interdependência, as quais também possuem o seu lado obscuro, nem sempre havendo a assimetria desejada ou imaginada. No quesito confiança é ímpar a busca do fortalecimento do capital social através de seus elementos - confiança, organização social, normas de reciprocidade, padrões de associativismo e redes - que por definição, ligam os vários segmentos da comunidade e valorizam costumes, relações de confiança e cooperação.

Por mais que se tenha dado maior enfoque para a interdependência colaborativa, AIO e trocas de conhecimento, entende-se que o tema relações interorganizacionais envolve muitas variáveis, além de ser complexo. Portanto, também ao se analisar os microprocessos nas relações interorganizacionais, todo o cuidado para não se realizar análises ingênuas faz-se necessário.

Finalizando retoma-se o que Barcelos, Eleutério e Giglio (2015) afirmam quanto à necessidade de se reconhecer a ausência de plataformas de investigação validadas, o que dificulta a construção de raciocínios, metodologias e instrumentos passíveis de utilização. Assim, apresentam-se algumas necessidades de pesquisa detectadas: 1) pesquisas sob diferentes perspectivas teóricas (defendemos nesse debate a abordagem sociológica), 2) mais pesquisas longitudinais e comparativas, 3) utilização de metodologias diversas de pesquisa, inclusive combinadas, 4) estudos que envolvam os microprocessos, 5) maior interação entre os pesquisadores do tema.

Sem a pretensão de apresentação de conclusões definitivas, ao se chegar ao final deste ensaio teórico acredita-se ter ampliado a perspectiva teórica e instigado debates mais aprofundados e pesquisas empíricas que trabalhem as relações interorganizacionais pela visão
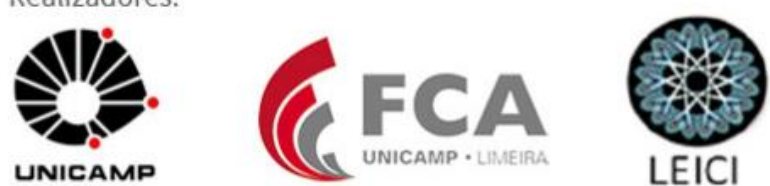
relacional da estratégia (perspectiva sociológica). Kirschbaum e Guarido Filho (2011, p.17) pontuam que "na perspectiva sociológica, os aspectos econômicos da ação estratégica ganham outros contornos". Acredita-se que tais contornos possam minimizar a derrocada que o capitalismo em crise impõe a sociedade. Portanto, ao se lançar luz a oportunidades alternativas de pesquisa, espera-se estar contribuindo com o entendimento da necessidade de reflexões e aprofundamento de questões essenciais nas pesquisas sobre a temática, não havendo a necessidade de aceitação do imposto pelo mainstream.

\section{Referências:}

AKTOUF, O. A administração entre a tradição e a renovação. Tradução: Roberto Fachin e Tânia Fischer. São Paulo: Atlas, 1996.

ANTONELLO, C. S.; GODOY, A. S. Aprendizagem organizacional no Brasil. Porto Alegre: Bookman, 2011.

BALESTRIN, A.; VERSCHOORE, J. Redes de cooperação empresarial: estratégias de gestão na nova economia. Porto Alegre: Bookman, 2008.

BALESTRIN, A.; VERSCHOORE, J.R.; PERUCIA, A. A visão relacional da estratégia: evidências empíricas em redes de cooperação empresarial. BASE - Revista de Administração e Contabilidade da Unisinos, v.11, n.1, p.47-58, 2014. DOI: 10.4013/base. 2014.111.04

BARCELOS, E. J. B. V.; ELEUTÉRIO, R. L.; GIGLIO, E. M. Análise crítica das contribuições das teses brasileiras sobre o tema de redes. Administração: ensino e pesquisa rio de janeiro, v. 16, n.1, p. 41-69, 2015.

BERTOLIN, R. V.; CAPPELLE, M. C. A.; BRITO, M. J. Corporeidade e estética na aprendizagem organizacional: insights emergentes. Revista de administração Mackenzie, v. 15, n. 2, mar./abr. 2014.

CROPPER, S.; EBERS, M.; HUXHAM, C.; RING, P. S. Handbook de relações interorganizacionais da Oxford. Porto Alegre: Bookman, 2014.

GIBB, J., SUNE, A., \& ALBERS, S. (2017). Network learning: Episodes of interorganizational learning towards a collective performance goal. European Management Journal, 35(1), 15-25.

GHERARDI, S. Practice-based theorizing on learning and knowing in organizations: an introduction. Organization, v.7, n.2, p. 211-23, 2000. Doi: 10.1177/135050840072001

GHERARDI, S. Introduction: the critical power of the practice lens. Management learning, v. 40, n. 2, p. 115-128, 2009a.

GHERARDI, S. Practice? it's a Matter of Taste! Management Learning, v. 40, n. 5, p. 535550, 2009b.
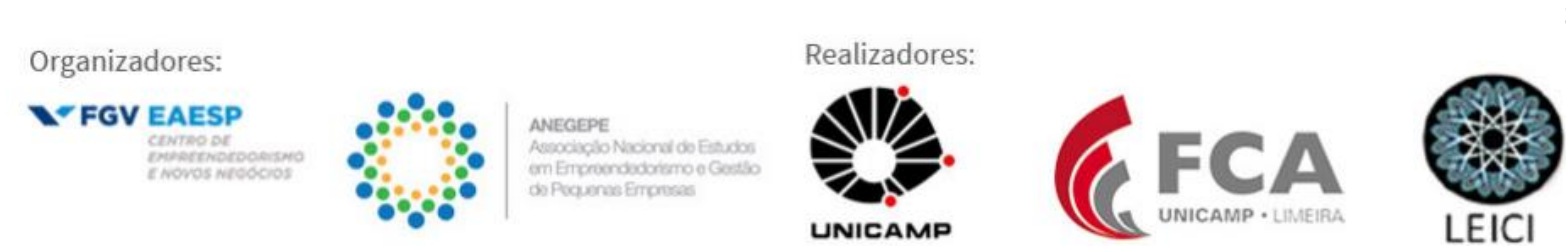
GHERARDI, S. Conhecimento situado e ação situada: o que os estudos baseados em prática prometem. In: GHERARDI, S.; STRATI, A. Administração e aprendizagem na prática. Rio de Janeiro: Elsevier, 2014.

GHERARDI, S. To start practice theorizing anew: The contribution of the concepts of agencement and formativeness. Organization, v. 1, n. 19, p. 1-19, 2015. Doi: $0.1177 / 1350508415605174$

JAPIASSU, H. O sonho transdisciplinar. In: COLÓQUIO INTERNACIONAL DE ESPISTEMOLOGIA E SOCIOLOGIA DA CIÊNCIA DA ADMINISTRAÇÃO, 3., 2013, Florianópolis. Conferência de abertura, Florianópolis, 2013. Documento não publicado.

KIRSCHBAUM, C. As Redes Intraorganizacionais são Inclusivas? Utopia e Testes. O\&S Salvador, v. 22, n. 74, p. 367-384, 2015.

KIRSCHBAUM, C.; GUARIDO FILHO, E. R. Perspectivas sociológicas da estratégia em organizações: uma introdução ao fórum, RAM, Revista Administração Mackenzie, v. 12, n. 6, p. 14-27, Edição Especial, 2011.

LUBATKIN, M.; FLORIN, J.; LANE, P. Learning together and apart: a model of reciprocal interfirm learning. Human Relations, v.54, n.10, p.1353-1382, 2001.

MATOS, M. P.; AMARAL FILHO, J.; COSTA, F.A. Arranjos e sistemas produtivos e inovativos culturais. In: MATOS, M. F.; CASSIOLATO, J. E.; LASTRES, H. M. M.;

LEMOS, C.; SZAPIRO, M. (Orgs). Arranjos produtivos locais: referencial, eperiências e políticas em 20 anos da RedeSist. Rio de Janeiro: E-Papers, 2017.

MOZZATO, A. R.; BITENCOURT, C. C. Understanding Interorganizational Learning Based on Social Spaces and Learning Episodes. BAR - Brazilian Administration Review, v.11, n.3, 2014.

MOZZATO, A. R.; GRZYBOVSKI, D. Abordagem crítica nos estudos organizacionais: concepção de indivíduo na perspectiva emancipatória. Cadernos EBAPE.BR (FGV), v. XI, p. 503-519, 2013.

MUTHUSAMY S. K.; WHITE, M. A. Learning and knowledge transfer in strategic alliances: a social exchange view. Organization Studies, v.26, n.3, p. 415-441, 2005.

PIRES, E. L. S. et al. Governança territorial: Conceito, fatos e modalidades. Rio Claro: Unesp-IGCE-Programa de pós-graduação em Geografia, 2011.

STRATI, A. Administração e aprendizagem na prática. Rio de Janeiro: Elsevier, 2014b. 
TENÓRIO, F. G. Tem razão a administração? Ensaios de teoria organizacional. 2. ed. Ijuí: Unijuí, 2004.

TENÓRIO, F. G. Pensamento crítico versus pensamento tradicional. Cadernos EBAPE.BR, v.7, n. 3, p. 472-491, set. 2009.

TURETA, C.; LIMA, J. B. Estratégia como prática social: o estrategizar em uma rede interorganizacional. RAM- Revista Administração Mackenzie, v. 12, n. 6, Edição Especial, 2011.

VERSCHOORE, J.R.; BULGACOV, S.; SEGATTO, A. P.; BATAGLIA, W. Concepções teóricas e verificações empíricas sobre a cooperação entre firmas no Brasil: uma introdução ao Fórum alianças estratégicas e redes de alianças. RAM, Revista Administração Mackenzie, v. 15, n. 3, p. 14-20, Edição Especial, 2014.

VIZEU, F.; GUARIDO FILHO, E. R.; GOMES, M. A. Para além do olhar econômico nas alianças estratégicas: implicações sociológicas do caso unihotéis. RAM- Revista Administração Mackenzie, v. 15, n.3, Edição Especial, p. 132-165, 2014. http://dx.doi.org/10.1590/1678-69712014

ZAHEER, A.; GÖZÜBÜYÜK, R.; MILANOV, H. It's the connections: the network perspective in the interorganizational research. Academy of Management Pespectives, v. 24, n.1, p.62-77, 2010.

SZAPIRO, M.; LEMOS, C.; LASTRES, H. M. M.; CASSIOLATO, J. E.; VARGAS, M. A. Panorama histórico da RedeSist e fundamentação teórica da abordagem de APL. In: MATOS, M. F.; CASSIOLATO, J. E.; LASTRES, H. M. M.; LEMOS, C.; SZAPIRO, M. (Orgs). Arranjos produtivos locais: referencial, eperiências e políticas em 20 anos da RedeSist. Rio de Janeiro: E-Papers, 2017. 